\title{
Role of Epidural Steroid Injection in Lumbar Spinal Stenosis-A Randomized Controlled Trial
}

\author{
Mohit Kishore Srivastava $\quad$ Anil Kumar Gupta ${ }^{1} \quad$ Sudhir R. Mishra ${ }^{1}$ Dileep Kumar ${ }^{1}$ Bal Krishna Ojha ${ }^{2}$ \\ Ganesh Yadav' \\ 1Department of Physical Medicine and Rehabilitation, King George's \\ Medical University, Lucknow, Uttar Pradesh, India \\ 2Department of Neurosurgery, King George's Medical University, \\ Lucknow, Uttar Pradesh, India \\ Address for correspondence Dileep Kumar, MS, Department \\ of Physical Medicine and Rehabilitation, King George's Medical \\ University, Shah Mina Road, Chowk, Lucknow 226003, \\ Uttar Pradesh, India (e-mail: rohitsriv14elec@gmail.com).
}

Indian J Neurosurg 2021;1:54-60.

\begin{abstract}
Keywords

- caudal

- epidural steroid

- degenerative lumbar spinal stenosis

Background Degenerative lumbar spinal stenosis (DLSS) is an important cause of pain and disability among the elderly and common indication for spinal surgery. However, due to age-related comorbidities, it becomes difficult for elderly patients of DLSS to immediately go for operative treatment. Caudal epidural steroid injection (CESI) can be an effective procedure for a selected group of patients who have chronic function-limiting lower back and lower extremity pain secondary to DLSS. The aim of this study was to compare the effects of CESI with physical therapy in patients afflicted with DLSS.

Materials and Methods It is a single center, open-label randomized controlled trial conducted in department of Physical Medicine and Rehabilitation at a tertiary care center of northern India from January 2016 to August 2017 among DLSS patients. Trial was registered under the clinical trial registry of India. Patients were randomized in two groups-32 in intervention group A (CESI with local anesthetic and physical therapy) and 32 in control group B (physical therapy alone). Outcome measures were numerical pain rating scale (NPRS), Oswestry disability index (ODI), and mean claudication distance (MCD) at $3,6,12$, and 24 weeks.

Results NPRS and ODI showed significant improvement at 3, 6, 12, and 24 weeks (group A > group B). Improvement in MCD was seen at each follow-up from baseline (group A > group B).

Conclusion Caudal epidural steroid administration can ameliorate pain, disability and claudication distance in DLSS patients, which provides them a window period for further definitive management.
\end{abstract}

\section{Introduction}

Degenerative lumbar spinal stenosis (DLSS) is one of the most debilitating health conditions among elderly individuals and the most recurrent indication for spinal surgery in individuals aged above 60 years. ${ }^{1}$ However, due to age-related comorbidities, it becomes difficult for elderly patients afflicted with DLSS to immediately go for operative treatment., ${ }^{2,3}$

DOI https://doi.org/ 10.1055/s-0040-1719234 ISSN 2277-954X.
Various conservative treatment modalities are being used to alleviate the symptoms of lumbosacral radiculopathy in patients with DLSS. Many elderly patients find conservative therapy appropriate, as it minimizes invasive intervention and decreases the risks of morbidity. ${ }^{4}$ In a study, it was reported that the conservative treatment for lumbar spinal stenosis is not a success for elderly patients, and they should be convinced to undergo operative treatment. ${ }^{5,6}$ Nandi et

(c) 2020. Neurological Surgeons' Society of India.

This is an open access article published by Thieme under the terms of the Creative Commons Attribution-NonDerivative-NonCommercial-License, permitting copying and reproduction so long as the original work is given appropriate credit. Contents may not be used for commercial purposes, or adapted, remixed, transformed or built upon. (https://creativecommons.org/licenses/by-nc-nd/4.0/)

Thieme Medical and Scientific Publishers Pvt. Ltd., A-12, 2nd Floor, Sector 2, Noida-201301 UP, India 
al reported that caudal epidural steroid injection (CESI) provides no additional improvement over placebo in the long-term natural history of lumbosacral radiculopathy. However, it can be important for short-term management of painful radiculopathy. ${ }^{7}$ Epidural injections may be considered as an effective procedure for a select group of patients who have chronic function-limiting lower back and lower extremity pain secondary to DLSS. ${ }^{8}$ So, this study aims to evaluate the overall short- and long-term effect of CESIs plus physical therapy in patients with DLSS as well as to compare its effect on pain scores and functional ability in patients receiving physical therapy alone.

\section{Materials and Methods}

\section{Study Design and Period}

The study was designed as a prospective, single-center, open-label, randomized controlled trial in accordance with the principles outlined in the Declaration of Helsinki and registered prospectively with the clinical trials registry, India (CTRI/2017/07/009036). Data recruitment and follow-up was done during the study period between January 2016 and August 2017.

\section{Study Setting}

The study was conducted in the Department of Physical Medicine and Rehabilitation at a tertiary care center of north India.

\section{Study Participants and Inclusion Criteria}

All patients of age 50 years or older with clinically and radiologically confirmed diagnosis of DLSS, having neurogenic claudication, with numerical pain rating scale (NPRS) score of more than 4, T2-weighted MRI showing lumbar midsagittal anteroposterior canal diameter at the most stenotic level as $\geq 13 \mathrm{~mm}$ and consented to participate were included in the study.

\section{Exclusion Criteria}

Patients with lateral recess or foraminal stenosis without the presence of central canal stenosis, with progressive neurological deficit or worsening neurological status/severe nerve root compression, primary or secondary malignancy of lumbar spine, vascular claudication, Pott's spine, peripheral neuropathy, history of prior surgery or fracture of lumbar spine, hip disease, severe peptic ulcer disease, bleeding tendencies, uncontrolled diabetes, pregnant or lactating mothers, past history of treatment with epidural steroid injections, psychiatric or cognitive problems that may hamper the outcome evaluation, and had active local/systemic infection were excluded.

Among the total 76 patients enrolled in the study, those excluded were as follows: three patients who had moderate-to-severe lumbar canal stenosis on MRI (midsagittal anteroposterior canal diameter $<13 \mathrm{~mm}$ ), two who had history treatment with epidural steroid injection, three patients who had pure foraminal stenosis without central canal stenosis, two patients who had avascular necrosis of hip joint, and two who had raised leucocyte count (suggestive of infection). A total of 64 patients were randomly allocated in the intervention and control group. The study design and participant flow chart are given in - Fig. 1 .

\section{Sample Size}

The sample size $(n)$ was determined using the following formula: $n=\left([Z \alpha / 2+Z \beta]^{2 *} 2^{*} \sigma^{2}\right) / \mathrm{d}$. ${ }^{2}$ Taking critical value $Z \alpha / 2$ as 1.96 , and $Z \beta$ as 0.84 and to detect hypothesized difference of 1.5 between the two groups (variants $=4.45$ ), significant with $95 \%$ confidence interval and power of $80 \%$, the required sample size was 32 patients in each group. ${ }^{9}$

\section{Patient Groups, Randomization, and Blinding}

All patients attending the day care facility of the hospital with clinical diagnosis of degenerative lumbar spinal stenosis were enrolled in the study. After recording the demographic data, they were subjected to detailed history and clinical examination. Serological tests included complete blood count, blood sugar (fasting and postprandial), C-reactive protein, serum levels of calcium, phosphate and alkaline phosphatase. This was followed by a radiological examination which included digital roentgenogram of lumbar spine and MRI of lumbar spine for assessment of diameter of spinal canal. MRI revealed DLSS as interpreted by a single radiologist. Canal stenosis was classified based upon midsagittal diameter measured in millimeters $(\mathrm{mm})$ obtained on T2 sagittal images at the narrowest stenotic level. The classification was mild $\geq 13 \mathrm{~mm}$, moderate $11-<13 \mathrm{~mm}$, and severe $\leq 11 \mathrm{~mm}$ diameter. The level with the most severe stenosis was used for the classification of the 64 patients: 39 had single level stenosis and 25 had multiple level stenosis. ${ }^{10}$ Baseline clinical outcome parameters, that is, NPRS, Oswestry disability index (ODI), and mean claudication distance (MCD) in feet were recorded. ${ }^{10-13}$

The patients were randomly allocated into two groups, that is, intervention and control group A and B, respectively. A computerized randomization table was created by a researcher who was not involved in the study. Each randomized patient was issued a concealed envelope with details of either form of treatment. The exact details of the treatment allocation of patients were concealed in an encrypted folder of the computer system. In the intervention group, CESI was administered to the study subjects by a trained clinician and this was followed by exercise program. The control group was only subjected to a planned physical therapy protocol under the supervision of a physical therapist who was unaware of the study. The follow-up and assessment of outcome was blinded, as it was done by clinicians who were not a part of the study. Every effort was made to minimize any form of bias in the study.

\section{Interventions}

Patients were given fluoroscopically guided CESI using $10 \mathrm{~mL}$ (0.5\%) Lignocaine with $80 \mathrm{mg}(2 \mathrm{~mL})$ Triamcinolone Acetonide in intervention group A. CESI was given by a trained clinician using Allengers C-arm HF49R. For confirmation of a successful block, relief of pain by at least $50 \%$, anesthesia or diminished sensation in distribution of blocked nerves was considered. After injection, patients were monitored to ensure stability 


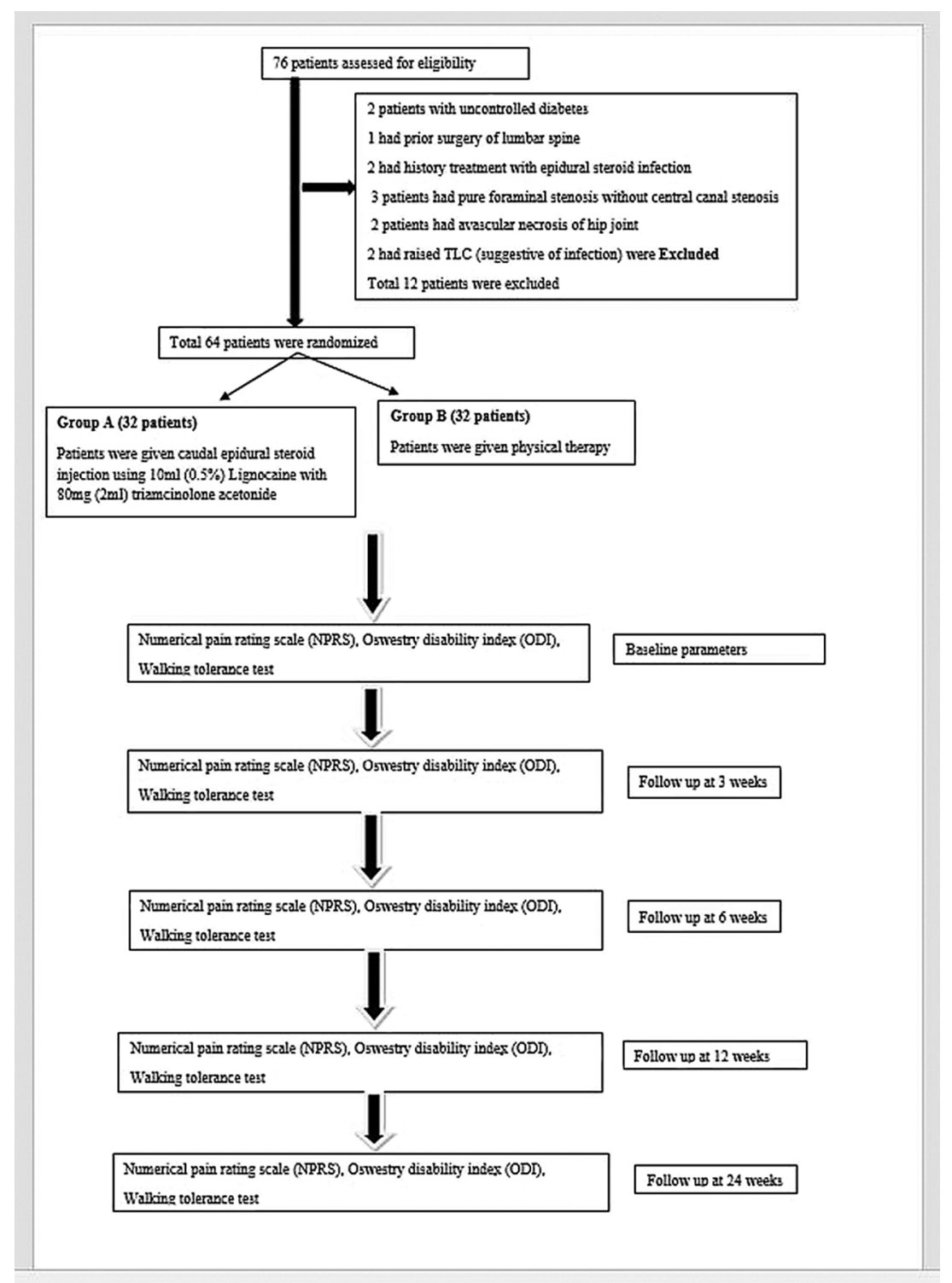

Fig. 1 Study design and participant flow.

of cardiovascular and respiratory systems. On aggravation of symptoms like pain in back, buttocks or legs, analgesics like diclofenac tablet $50 \mathrm{mg}$ was prescribed SOS. Similar physical therapy protocol was given in the intervention group as was in the control group.

In control group $\mathrm{B}$, only physical therapy was given. It included three sets of 10 posterior pelvic tilts, two sets of single and double knee to chest and self-lumbar side exercises and, finally, three sets of 30 seconds for specific hip flexor stretching for rectus femoris and iliopsoas. Transversus abdominis isometric exercises were also done. Supervised physical therapy was done for 5 days a week, that is, a total of 15 sessions were given. Patients were constantly motivated for completing the 15 sessions, and if one session was missed, it was scheduled on other days. After completing the protocol, patients were advised to continue physical therapy at home also. Details of home-based physical therapy was confirmed on telephone by the investigator once a week in both the groups during the study period.
No serious complications like epidural abscess, infection or haematoma were reported in the intervention group during the study period of 6 months.

\section{Statistical Analysis}

The data was analyzed using SPSS version 24.0. Descriptive statistics were calculated using mean, standard deviation (SD), percentages, column graphs and cross-tabulations. Intra- and intergroup analysis was done by comparing mean using repeated measures Anova and independent $t$-test, respectively. Probability $(p)$ was calculated to test statistical significance at the $5 \%$ level of significance. $p<0.05$ was considered as statistically significant.

\section{Results}

A total of 64 subjects were randomized into two groups for study. The mean age of group A was $60.66 \pm 7.29$ years, and in group $B$, it was $61.13 \pm 7.03$ years. No significant 
difference ( $p=0.794$ ) was found between the average ages of the two groups. The male:female ratio in group $\mathrm{A}$ was 59.4: 40.6 percent, while in group, B it was 40.6:59.4 percent. No significant difference $(p=0.134)$ was found between the sex ratio of the two groups. As much as 62.8 percent of group A and 60.2 percent of group B participants had single-level stenosis, while 70.6 percent of group A and 68.3 percent of group $B$ had grade $B$ level of stenosis, and there was no significant difference. The mean midsagittal canal diameter was $14.3 \pm 0.52 \mathrm{~mm}$ and $14.7 \pm 1.08 \mathrm{~mm}$ in group A and B, respectively, and there was no significant mean difference in the diameter in the intervention and control group (-Table 1).

\section{Comparison of NPRS at Various Follow-ups}

In the intragroup comparison of NPRS of group A among various follow ups, it was found that the mean NPRS was found to be $8.78 \pm 0.02$ at baseline, which was reduced to $3.78 \pm 0.98$ in the 3 weeks follow-up, $5.01 \pm 0.88$ in the 6 weeks follow-up and that was maintained to $4.43 \pm 1.11$ in the 12 weeks follow-up, and finally it was $4.49 \pm 0.25$ in the 24 weeks follow-up. Similarly, in group B, mean NPRS was found to be $8.90 \pm 0.12$ at baseline, which was reduced to $7.34 \pm 0.87$ in the 3 weeks follow-up, further reduced to $6.61 \pm 1.04$ in the 6 weeks follow-up, $5.98 \pm 0.39$ in the 12 weeks follow-up, and finally it was $5.74 \pm 0.44$ in the
24 weeks follow-up. The reduction in mean NPRS from baseline to last follow-up in both group A and B was highly significant $(p<0.001)$.

In the intergroup comparison, statistically significant difference $(p<0.001)$ was observed in mean NPRS at 3, 6, 12 , and 24 weeks, respectively, but mean NPRS of group A was less than group B throughout, thereby the intervention group showed better improvement than control group (-Table 2).

\section{Comparison of ODI at Various Follow-ups}

In the intragroup comparison of size of ODI (\%) of group A among various follow-ups, it was found that the mean ODI was found to be $71.06 \pm 7.87$ at baseline which was reduced to $64.31 \pm 7.07$ in the 3 weeks follow-up, to $57.25 \pm 7.19$ in the 6 weeks follow-up, to $56.03 \pm 7.16$ in 12 weeks follow-up, and finally it was $55.01 \pm 5.24$ in the 24 weeks follow-up. Similarly, in group $B$, the mean ODI was $70.19 \pm 8.64$ baseline which was reduced to $68.69 \pm 8.71$ in the 3 weeks follow-up, to $61.44 \pm 8.21$ in the 6 weeks follow-up, to $60.31 \pm 7.67$ in the 12 weeks follow-up, and finally it was $59.28 \pm 6.11$ in the 24 weeks follow-up. The reduction in mean ODI from baseline to last follow-up in both group A and B was highly significant ( $p<0.001)$.

In the intergroup comparison, statistically significant difference $(p<0.05)$ was observed in mean ODI at 3 weeks,

Table 1 Baseline characteristics of study participants in intervention and control group

\begin{tabular}{|l|l|l|l|l|}
\hline \multicolumn{2}{|l|}{ Characteristics } & $\begin{array}{l}\text { Group A } \\
(\boldsymbol{n}=32)\end{array}$ & $\begin{array}{l}\text { Group B } \\
(\boldsymbol{n}=32)\end{array}$ & $p$-Value \\
\hline Age (years) & Mean \pm SD & $60.66 \pm 7.29$ & $61.13 \pm 7.03$ & $0.794^{\mathrm{a}}$ \\
\hline \multirow{2}{*}{ Sex } & Males & $19(59.4)$ & $13(40.6)$ & $0.134^{\mathrm{b}}$ \\
\cline { 2 - 5 } & Females & $13(40.6)$ & $19(59.4)$ & $0.066^{\mathrm{b}}$ \\
\hline \multirow{2}{*}{ Level of stenosis } & Single level & $20(62.8)$ & $19(60.2)$ & $13(39.8)$ \\
\cline { 2 - 5 } & Multiple level & $12(37.2)$ & $14.7 \pm 1.08$ & $0.064^{\mathrm{a}}$ \\
\hline Midsagittal canal diameter $(\mathrm{mm})$ & Mean \pm SD & $14.3 \pm 0.52$ & & \\
\hline
\end{tabular}

Note: At the time of patient enrolment, number (percentage).

andependent $t$ test.

${ }^{b}$ Chi square test

Table 2 Between and within group comparison of NPRS at various follow-ups

\begin{tabular}{|c|c|c|c|c|}
\hline \multirow[t]{2}{*}{ Follow up time } & \multicolumn{2}{|c|}{ NPRS } & \multirow[t]{2}{*}{$t$ value } & \multirow[t]{2}{*}{$p$-Value ${ }^{a}$} \\
\hline & $\begin{array}{l}\text { Group A } \\
(n=32)\end{array}$ & $\begin{array}{l}\text { Group B } \\
(n=32)\end{array}$ & & \\
\hline Baseline & $8.78 \pm 0.02$ & $8.90 \pm 0.12$ & 5.580 & $<0.001^{b}$ \\
\hline 3 weeks & $3.78 \pm 0.98$ & $7.34 \pm 0.87$ & 15.367 & $<0.001^{\mathrm{b}}$ \\
\hline 6 weeks & $5.01 \pm 0.88$ & $6.61 \pm 1.04$ & 6.644 & $<0.001^{\mathrm{b}}$ \\
\hline 12 weeks & $4.43 \pm 1.11$ & $5.98 \pm 0.39$ & 7.453 & $<0.001^{b}$ \\
\hline 24 weeks & $4.49 \pm 0.25$ & $5.74 \pm 0.44$ & 13.973 & $<0.0001^{b}$ \\
\hline$p$-Value ${ }^{c}$ & $<0.001$ & $<0.001$ & & \\
\hline Multiple comparisons from baseline $(p<0.01)$ & All pairs & All pairs & & \\
\hline
\end{tabular}

Abbreviation: NPRS, numerical pain rating scale.

andependent $t$ test.

${ }^{\mathrm{b}} \mathrm{p}<0.01$.

'Repeated measures Anova test. 
Table 3 Between and within group comparison of ODI at various follow-ups

\begin{tabular}{|c|c|c|c|c|}
\hline \multirow[t]{2}{*}{ Follow-up time } & \multicolumn{2}{|c|}{ ODI } & \multirow[t]{2}{*}{$t$ value } & \multirow[t]{2}{*}{$p$-Value ${ }^{a}$} \\
\hline & $\begin{array}{l}\text { Group A } \\
(n=32)\end{array}$ & $\begin{array}{l}\text { Group B } \\
(n=32)\end{array}$ & & \\
\hline Baseline & $71.06 \pm 7.87$ & $70.19 \pm 8.64$ & -0.421 & 0.675 \\
\hline 3 weeks & $64.31 \pm 7.07$ & $68.69 \pm 8.71$ & 2.209 & $0.031^{b}$ \\
\hline 6 weeks & $57.25 \pm 7.19$ & $61.44 \pm 8.21$ & 2.172 & $0.033^{b}$ \\
\hline 12 weeks & $56.03 \pm 7.16$ & $60.31 \pm 7.67$ & 2.307 & $0.024^{b}$ \\
\hline 24 weeks & $55.01 \pm 5.24$ & $59.28 \pm 6.11$ & 3.001 & $0.004^{b}$ \\
\hline$p$-Value ${ }^{c}$ & $<0.001$ & $<0.001$ & & \\
\hline Multiple comparisons from baseline & All pairs & All pairs & & \\
\hline
\end{tabular}

Abbreviation: ODI, Oswestry Disability Index.

andependent $t$ test.

${ }^{\mathrm{b}} p<0.05$ is significant.

'Repeated measures Anova test.

6 weeks and 12 weeks, respectively, and mean ODI of group A was less than group B throughout, thereby the intervention group showed better improvement than control group (-Table 3).

\section{Comparison of Mean Claudication Distance (MCD) in group $A$ and $B$}

The MCD in group A and B was 457 and 294 feet, respectively, at 3 weeks. Similarly, it was 622 feet in group A and 453 feet in group $B$ at 6 weeks. Further at 12 weeks, the MCD was 671 and 476 feet in group A and B, respectively. There was a significant difference in MCD at 3,6 , and 12 weeks ( $p$ $<0.0001$ ) (-Fig. 2).

\section{Discussion}

With increasing longevity of life and aging populations, the prevalence and associated clinical disability related to degenerative lumbar spinal stenosis is on the rise. The results of the study show a clear benefit from the use of CESIs using Lignocaine and Triamcinolone acetonide along with physical therapy in patients with DLSS. There was statistically and clinically significant reduction in pain and disability in the intervention group. This benefit was seen after the injection at 3,6, 12 and 24 weeks when compared with physical therapy alone.

In our study, majority of the patients of DLSS were males. Similar male predominance was depicted by Cohen et al, ${ }^{14} \mathrm{Koc}$ et $\mathrm{al}^{15}$ and many other researchers. ${ }^{6,7,16,17}$ This is in contrast to study conducted by Botwinet $\mathrm{al}^{10}{ }^{10}$ Delport et $\mathrm{al},{ }^{18}$ Southern et $\mathrm{al}^{19}$ where female predominance was seen. There seems to be a change in trend of DLSS as per gender, and a shift is seen from females to males in the past 5 years. This is attributed to the fact that there is increase in incidence of male obesity due to changes in food habits which is more central and can result in pathologies of spine. ${ }^{20}$

We observed a significant difference in improvement of pain at 3, 6, 12 and 24 weeks in patients receiving CESIs, which is similar to Nandi et $\mathrm{al}^{,}{ }^{8} \mathrm{Koc}$ et $\mathrm{al}^{15}$ and Arden et $\mathrm{al}^{17}$ who also found a statistically significant

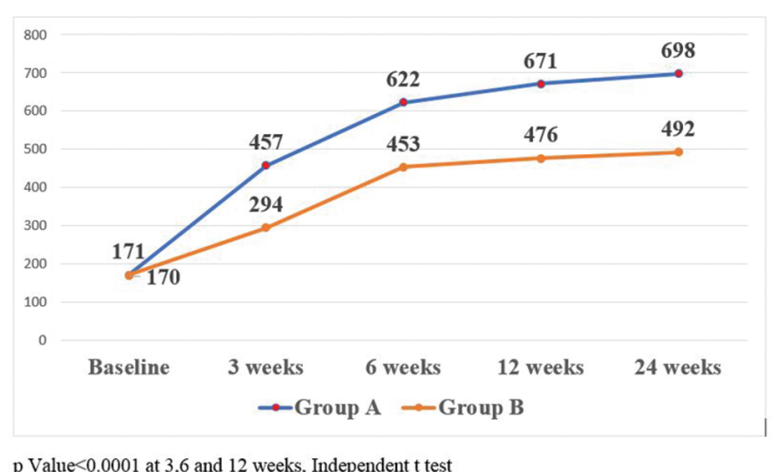

$\mathrm{p}$ Value $<0.0001$ at 3.6 and 12 weeks. Independent $t$ test

Fig. 2 Mean claudication distance (MCD) (feet) by walking tolerance test at various follow-ups.

improvement in pain at 3 weeks in patients receiving CESIs, followed by no benefit from 6 to 52 weeks. Evidencebased, short-term efficacy of CESIs in ameliorating pain by more than 50 percent has been reported by many studies. ${ }^{13,21,22}$ In contrast to this, few researchers have observed no significant improvement in pain at 12 weeks in patients with DLSS. They have explained that low-efficacy, underdosage, and a dilution effect due to the high-volumes injected could have influenced the effect of CESIs in their study. ${ }^{14,23}$

Our study reports a significant difference in improvement of disability at 3, 6, 12 and 24 weeks after CESIs, indicating both short- and long-term improvement in DLSS. Our results are supported by Radcliffe et al, ${ }^{16}$ as they showed a gradual improvement in disability with respect to duration from the day of receiving the CESIs, with a peak at 24 weeks and decline after that. Koc et a ${ }^{15}$ compared the efficacy of CESIs with physical therapy and placebo and reported significant improvement in disability at 6 months in all the three groups from baseline. Our findings are in agreement with other studies also. ${ }^{21,22}$

Our findings of NPRS and ODI at 3, 6, 12 and 24 weeks are in concordance with Botwin et al ${ }^{13}$ who has reported in their study an improvement in pain and disability at 6 months. 
Based on a systematic review on CESIs in 2015, the evidence is Level II for long-term, 2-year efficacy for CESIs with local anesthetic alone or with local anesthetic and steroids. ${ }^{24}$

Many systematic reviews have been conducted to assess the efficacy of CESIs for disability improvement, as there is a lot of discrepancy regarding their short-term or long-term benefits. ${ }^{24-26}$ This is attributed to the fact that improvement in disability is correlated with the age of the patient. Comorbid conditions play a major role in outcome of disability after the intervention, which is especially more pronounced in elder age groups. ${ }^{27}$

No significant difference was seen in the MCD between intervention and control group at 24 weeks. Our findings can be supported by the fact that the effect of steroids dissipates over time due to which none of the outcome measures were significant at 6 week follow-ups. ${ }^{14} \mathrm{~A}$ study by Huda et al ${ }^{28}$ also found a significant difference in walking tolerance test at 4 weeks in patients receiving CESIs but no difference at 12 weeks and that was statistically significant $(p<0.05)$.

The sociodemographic pattern, lifestyle of population, dietary habits, risk of comorbidity, and wide variation in consumption of tobacco and alcohol have a direct as well as an indirect effect on the status of pain and disability. This draws attention to the fact of availability of very low evidence about efficacy of CESIs in Indian population. Very few Indian studies have assessed the efficacy of CESIs in patients with DLSS; therefore, our study has highlighted compelling evidence regarding the same.

\section{Strengths and Limitations}

The strengths of our study is that it discloses new avenues for more research concerning the long-term efficacy of CESIs, especially in patients suffering with mild and moderate stenosis and those patients who are more than 50 years of age, as both CESIs with physical therapy and a well-planned exercise program seem to bring nearly same improvement in patients with DLSS at 24 weeks. There is a need for safer, less-invasive treatment options for older adults with DLSS for reducing their functional disability and promoting independence because older adults have comorbid conditions such as diabetes that may increase the risks due to systemic absorption of corticosteroids as compared with compared with young and middle-age adults.

Our study has the following limitations: First, due to the heterogeneous nature of the intervention, that is, caudal epidural steroid injection and physical therapy versus physical therapy, patients were aware of the intervention, so that might have altered their perception of pain. Second, a larger sample size would have helped in better generalization of our findings.

\section{Conclusion}

The study concludes that in case of mild canal compromise and mild-to-moderate compression of nerve roots, caudal epidural steroid injections along with physical therapy are helpful for alleviating pain and disability as compared with only physical therapy given alone, which provides these elderly patients a window period for undergoing any operative procedure in future, as this group shows poor patient compliance and delayed consent for surgical management, due to fear of loss of independence postsurgery and presence of various comorbidities in this age group. Moreover, only physical therapy requires motivation and inner strength on the part of the patient, so addition of caudal epidural steroid injection offers them additional benefit in terms of symptom relief.

\section{Ethical Approval}

The study was ethically approved by the Institutional Review Board of the University (1261/ethics/R.cell-16). Informed consent was requested and obtained from all patients enrolled in the study.

\section{Conflict of Interest}

None declared.

\section{Acknowledgment}

The author is highly grateful to Late Prof V. P. Sharma, Ex Professor and Head, Department of Physical Medicine and Rehabilitation, KGMU, Lucknow for his guidance and support.

\section{References}

1 Szpalski M, Gunzburg R. The role of surgery in the management of low back pain. Baillieres Clin Rheumatol 1998;12(1):141-159

2 Ferrero E, Lonjon G, Bouyer B, Sabourin M, Ould-Slimane M, Guigui P. Influence of comorbidities on patients reported outcomes in degenerative lumbar spinal stenosis. Orthop Traumatol Surg Res 2018;104(7):1031-1036

3 Slover J, Abdu WA, Hanscom B, Lurie J, Weinstein JN. Can condition-specific health surveys be specific to spine disease? An analysis of the effect of comorbidities on baseline condition-specific and general health survey scores. Spine 2006;31(11):1265-1271

4 Nagler W, Hausen HS. Conservative management of lumbar spinal stenosis. Identifying patients likely to do well without surgery. Postgrad Med 1998;103(4):69-71

5 Shabat S, Folman Y, Leitner Y, Fredman B, Gepstein R. Failure of conservative treatment for lumbar spinal stenosis in elderly patients. Arch Gerontol Geriatr 2007;44(3):235-241

6 Manchikanti L, Kaye AD, Manchikanti K, Boswell M, Pampati V, Hirsch J. Efficacy of epidural injections in the treatment of lumbar central spinal stenosis: a systematic review. Anesth Pain Med 2015;5(1):e23139

7 Nandi J, Chowdhery A. A randomized controlled clinical trial to determine the effectiveness of caudal epidural steroid injection in lumbosacral sciatica. J Clin Diagn Res 2017; 11(2):RC04-RC08

8 Lurie J, Tomkins-Lane C. Management of lumbar spinal stenosis. BMJ 2016;352:h6234

9 UK Select Statistics. Available at: https://select-statistics.co.uk/ calculators/sample-size-calculator-population-proportion. Accessed July 16, 2020

10 Botwin K, Brown LA, Fishman M, Rao S. Fluoroscopically guided caudal epidural steroid injections in degenerative lumbar spine stenosis. Pain Physician 2007;10(4):547-558

11 Kao SC, Lin CS. Caudal epidural block: an updated review of anatomy and techniques. BioMed Res Int 2017;2017:9217145

12 McCaffery M, Pasero C. Teaching patients to use a numerical pain-rating scale. Am J Nurs 1999;99(12):22

13 Fairbank JC, Pynsent PB. The Oswestry disability index. Spine 2000;25(22):2940-2952

14 Cohen SP, Hanling S, Bicket MC, et al. Epidural steroid injections compared with gabapentin for lumbosacral radicular 
pain: multicenter randomized double blind comparative efficacy study. BMJ 2015;350:h1748

15 Koc Z, Ozcakir S, Sivrioglu K, Gurbet A, Kucukoglu S. Effectiveness of physical therapy and epidural steroid injections in lumbar spinal stenosis. Spine 2009;34(10):985-989

16 Radcliff K, Kepler C, Hilibrand A, et al. Epidural steroid injections are associated with less improvement in the treatment of lumbar spinal stenosis: a subgroup analysis of the SPORT. Spine 2013;38:279

17 Arden NK, Price C, Reading I, et al; WEST Study Group. A multicentre randomized controlled trial of epidural corticosteroid injections for sciatica: the WEST study. Rheumatology (Oxford) 2005;44(11):1399-1406

18 Delport EG, Cucuzzella AR, Marley JK, Pruitt CM, Fisher JR. Treatment of lumbar spinal stenosis with epidural steroid injections: a retrospective outcome study. Arch Phys Med Rehabil 2004;85(3):479-484

19 Southern D, Lutz GE, Cooper G, Barre L. Are fluoroscopic caudal epidural steroid injections effective for managing chronic low back pain? Pain Physician 2003;6(2):167-172

20 Knutsson B, Sandén B, Sjödén G, Järvholm B, Michaëlsson K. Body mass index and risk for clinical lumbar spinal stenosis. Spine 2015;40(18):1451-1456

21 Manchikanti L, Cash KA, McManus CD, Pampati V, Smith HS. One-year results of a randomized, double-blind, active controlled trial of fluoroscopic caudal epidural injections with or without steroids in managing chronic discogenic low back pain without disc herniation or radiculitis. Pain Physician 2011; $14(1): 25-36$
22 Barré L, Lutz GE, Southern D, Cooper G. Fluoroscopically guided caudal epidural steroid injections for lumbar spinal stenosis: a retrospective evaluation of long term efficacy. Pain Physician 2004;7(2):187-193

23 Iversen T, Solberg TK, Romner B, et al. Effect of caudal epidural steroid or saline injection in chronic lumbar radiculopathy: multicentre, blinded, randomised controlled trial. BMJ 2011;343:d5278

24 Manchikanti L, Nampiaparampil DE, Manchikanti KN, et al. Comparison of the efficacy of saline, local anesthetics, and steroids in epidural and facet joint injections for the management of spinal pain: A systematic review of randomized controlled trials. Surg Neurol Int 2015;6(Suppl 4):S194-S235

25 Parr AT, Diwan S, Abdi S. Lumbar interlaminar epidural injections in managing chronic low back and lower extremity pain: a systematic review. Pain Physician 2009;12(1):163-188

26 Parr AT, Manchikanti L, Hameed H, et al. Caudal epidural injections in the management of chronic low back pain: a systematic appraisal of the literature. Pain Physician 2012; 15(3):E159-E198

27 Heiland EG, Welmer AK, Wang R, et al. Association of mobility limitations with incident disability among older adults: a population-based study. Age Ageing 2016;45(6):812-819

28 Huda N, Bansal P, Gupta SM, et al. The efficacy of epidural depo-methylprednisolone and triamcinolone acetate in relieving the symptoms of lumbar canal stenosis: A comparative study. J Clin Diagn Res 2010;4:2842-2847 